\title{
Un análisis empírico de la financiación de la pyme metalmecánica del Valle del Cauca: 2000 -2006*
}

\section{AN EMPIRICAL ANALYSIS OF THE FINANCING OF METALLURGICAL SMES OF Valle del Cauca: 2000 -2006}

Jorge Alberto Rivera Godoy*

\section{RESUMEN}

Esta investigación identifica la estructura financiera y los factores explicativos de la estructura de capital de las pymes vallecaucanas del sector metalmecánica en el período 2000 -2006.

Se tiene como soporte las principales teorías sobre la estructura de capital y sus pruebas de campo en el ámbito internacional y nacional, y se utiliza como metodología el análisis económico - financiero y un modelo econométrico de Datos de Panel.

El estudio encuentra diferencias en la estructura financiera y en los factores que explican el endeudamiento a largo plazo de la pequeña, mediana y pyme, a pesar de la similitud de su actividad y de pertenecer a una misma región.

La pequeña se endeuda más y la mediana concentra su deuda a corto plazo.

Los factores determinantes de la estructura de capital son: endeudamiento a corto plazo, volatilidad de la utilidad operacional, protección fiscal diferente a la deuda, oportunidad de crecimiento, rentabilidad, activos tangibles, tamaño y edad de la firma.
ABSTRACT

This research identifies the financial structure and the factors that explain the capital structure of vallecaucanas SMEs of the metallurgical sector in the period $2000-2006$.

Supported by the main theories about the capital structure and its field trials at the international and national scope, and is used as metodhology the economic-finance analysis, and an econometric model of Data Panel.

The study found differences in the financial structure and the factors that explain the longterm debt of the small, medium and SME, despite the similarity of their activity and belonging to the same region.

The small company gets in more debt and the medium company concentrates its short-term debt.

The determinants of capital structure are: short term debt, volatility of the operational utility, tax protection different from debt, growth opportunity, profitability, tangible assets, size and age of the firm.

Filósofo, de la Universidad de Antioquia. Especialista en Gobierno y Cultura Política, de la Universidad de Antioquia. Obtuvo el DEA en 2004. Candidato a Doctor en filosofía política en la Universidad de Granada.

Actualmente, Docente de la Escuela de Ciencias Estratégicas de la Universidad Pontificia Bolivariana de Medellín e investigador del Grupo Estudios Empresariales de la Facultad de Administración de la Universidad Pontificia Bolivariana de Medellín - Colombia. Correo Electrónico: nicolas.molina@upb.edu.co

Articulo Tipo 1: de Investigación Cientifica y Tecnológica. Según clasificación de Colciencias.

Fecha de Recepción: 15 de junio del 2007 
Al cotejar los resultados con modelos teóricos y evidencia empíricas, no se prueba que se ajuste a alguno de ellos; lo que promueve a generar investigaciones más profundas sobre cada uno de los factores determinantes de la estructura de capital.

Palabras claves: Financiación empresarial, estructura de capital, determinantes del endeudamiento, Pyme, sector metalmecánica.
By collating the results with theoretical models and empirical evidence there is no proof that it meets any of them; this promotes to generate deeper research on each of the determinants of capital structure.

Keywords: corporate financing, capital structure, determinants of indebtedness, SME, metallurgical industry.

\section{INTRODUCCIÓN}

Para saber si la gestión de financiación de las pyme metalmecánica ubicada en el Valle del Cauca influye sobre el valor de la empresa, se hace necesario investigar las decisiones de financiación de la empresa, ¿cuál es su estructura de capital?, ¿qué factores influyen en estas decisiones? y ¿a qué modelos teóricos o evidencias empíricas se ajustan?

En este estudio se aborda estas inquietudes siguiendo la siguiente secuencia: 1. Generalidades del sector metalmecánica. 2. Marco teórico 3. Marco de referencia. 4. Estudio de campo de las pymes del sector metalmecánica del Valle del Cauca. 4.1 Estructura financiera. 4.2 Factores explicativos de la estructura de capital. 5. Análisis y comparación de resultados. 6. Conclusiones

\section{El SECTOR METALMECÁNICA}

Aproximadamente el 5\% del total de establecimientos industriales en Colombia es del sector metalmecánica, conformado en un $79,1 \%$ por pymes, $16,4 \%$ por grandes empresas y el otro $4,4 \%$ por micros. De las 1.052 empresas industriales localizadas en el Valle del Cauca, 43 se dedican a la actividad metalmecánica, es decir el $4,1 \%$; siendo el $79,1 \%$ pyme y el $20,9 \%$ restante gran empresa. Ver cuadro 1.

El sector metalmecánica contribuye con el 5,2\% del PIB que crea la industria en Colombia, y genera el $4 \%$ de su empleo. En el Valle del Cauca este sector aporta el $8 \%$ del PIB industrial de la región. Ver cuadro 2.

CUADRO No. 1

Establecimientos Industriales

\begin{tabular}{|c|c|c|c|c|c|c|c|c|c|c|c|c|}
\hline \multirow{2}{*}{ Establecimientos } & \multicolumn{2}{|c|}{ Micros } & \multicolumn{2}{|c|}{ Pequeña } & \multicolumn{2}{|c|}{ Medianas } & \multicolumn{2}{|c|}{ Pymes } & \multicolumn{2}{|c|}{ Grandes } & \multicolumn{2}{|c|}{ Total } \\
\hline & & & No. & $\%$ & No. & $\%$ & No. & $\%$ & No. & $\%$ & No. & $\%$ \\
\hline Industriales & 138 & 1,8 & 1.546 & 20,6 & 2.320 & 30,8 & 3.866 & 51,4 & 3.520 & 46,8 & 7.524 & 100 \\
\hline $\begin{array}{l}\text { Metalinecánica } \\
\text { Valle del Cauca }\end{array}$ & 17 & 4,4 & 186 & 48,6 & 117 & 30,5 & 303 & 79,1 & 63 & 16,4 & 383 & 100 \\
\hline Industriales & 17 & 1,6 & 221 & 21 & 225 & 21,4 & 446 & 42,4 & 589 & 56 & 1.052 & 100 \\
\hline Metalmecánica & 0 & 0 & 19 & 44,2 & 15 & 34,9 & 34 & 79,1 & 9 & 20,9 & 43 & 100 \\
\hline
\end{tabular}

FUENTE: Elaboración propia, con información extraida de la Superintendencia de Sociedades (2007a y 2007b), DANE (2007)

CUADRO No. 2

PIB y empleo

\begin{tabular}{l|r|r|r|r}
\hline & PIB (en miles \$) & $\%$ PIB & Empleo & $\%$ Empleo \\
\hline Nacional & 108.340 .147 .335 & 100,0 & 587.630 & 100,0 \\
Industria & 5.649 .861 .810 & 5,2 & 23.479 & 4,0 \\
Metalinecánica & & & & \\
Valle del Cauca & 15.336 .228 .830 & 100,0 & 83.265 & \\
Industria & 1.259 .031 .162 & 8,2 & & \\
Metalmecánica &
\end{tabular}

FUENTE: elaboración propia, con información extraída de Superintendencia de Sociedades (2007a), DANE (2007). 


\section{Marco teórico}

Desde mediados del siglo pasado Miller y Modigliani (1958) dieron inicio al debate si la forma como las empresas financiaban sus actividades operativas generaba, o no valor a la empresa. Sus preliminares conclusiones de que el valor de una empresa apalancada, $\mathrm{Vl}$, es semejante a una no apalancada, $\mathrm{Vu}$, hasta la fecha no ha tenido respuesta concluyente, pero si ha promovido el desarrollo de numerosas teorías que pretende poner a consideración algunos factores que influyen sobre la decisión de endeudamiento.

Aunque la discusión se inició bajo escenarios de mercados perfectos, sin considerar los impuestos, diferentes propuestas teóricas han evaluado el impacto sobre el valor de empresa incluyendo en sus modelos, de forma parcial, el impuesto de sociedades, otras protecciones fiscales diferentes a la deuda e imperfecciones del mercado como los costos de dificultades financieras, los costos de agencia y la información asimétrica.
En el cuadro No. 3 se presenta los modelos teóricos que pretenden mostrar que existe una combinación ideal de deuda-capital que maximiza el valor de la empresa, una vez que se equilibren los beneficios y costos de la deuda, se conocen en el medio académico como la teoría del trade off. (Balance de los costos y beneficios). Aunque en la columna de la izquierda se acumula sus efectos sobre el valor de la empresa, no existe un macro-modelo que los integre.

En contraposición a esta teoría ha surgido la teoría del pecking order (jerarquía de preferencia) que no comparte la tesis de que exista una razón de endeudamiento óptima, porque la política de financiación de la empresa privilegia el uso de recursos que menos problema forme por la asimetría de información entre directivos e inversores externos; siguiéndose una preferencia de recursos en este orden: primero, fondos internos, después deuda, y por último acciones debido a la señal negativa que genera al mercado.

CUADRO No. 3

Principales modelos teóricos que relacionan el endeudamiento con el valor de la empresa.

\begin{tabular}{l|l}
\hline Modelo teorico sobre la estructura de capital & Valor de la empresa endeudada (V1) \\
\hline $\begin{array}{l}\text { Miller y Modigliani (1963), incluyen los impuestos de } \\
\text { sociedades, concluyando que genera una ventaja fiscal por } \\
\text { deuda tD }\end{array}$ & $V_{1}=V_{u}+t D$ \\
\hline $\begin{array}{l}\text { Deangelo y Masulis (1980) muestran que existe otros ahorros } \\
\text { diferentes a la deuda por lo tanto } D \text { se convierte en } t D_{n}\end{array}$ & $V_{1}=V_{u}+t D_{n}$ \\
\hline $\begin{array}{l}\text { Altman (1984) y Kim (1978) consideran los cortos de quiebra } \\
\text { en funcion del endeudamiento } Q(D / V)\end{array}$ & $V_{1}=V_{u}+t D_{n}-Q(D / V)$ \\
\hline $\begin{array}{l}\text { Jense y Meckling (1976) contemplan la relación entre la } \\
\text { finaciación y los costos de agencia } A_{d}+A_{s}\end{array}$ & $V_{1}=V_{u}+\left[t D_{n}-Q(D / V)-\left(A_{s}+A_{d}\right)\right]$ \\
\hline $\begin{array}{l}\text { Ross (1977) y Ravid y Saring (1991) muestran que en escenario } \\
\text { de información asimetrica, emitir deuda S } S_{d} \text { es una señal } \\
\text { positiva, mientras es una señal negativa emitir acciones } S_{s}\end{array}$ & $V_{1}=V_{u}+\left[t D_{n}-Q(D / V)-\left(A_{s}+A_{d}\right)+\left(S_{d}-S_{s}\right)\right]$ \\
\hline
\end{tabular}

Convenciones: $t$ es la tasa de impuesto de la sociedad. D es el valor de mercado de la deuda. V es el valor de mercado de la empresa. tDn es la ventaja impositiva por deuda reducida por protecciones fiscales alternas. Q (D / V) son los costos por dificultades financieras en función creciente del ratio de endeudamiento. Ad As son los costos de agencia por la emisión de deuda y con acciones respectivamente. Sd Ss son los efectos sobre el valor de la empresa por emitir deuda y acciones respectivamente.

FUENTE: Elaboración propia.

Harris y Raviv (1991) identifican, clasifican y asocian algunos factores explicativos de acuerdo a las más recientes teorías sobre la estructura de capital; algunos de más relevantes se muestra en el cuadro 4. Esta expansión de variables explicativas puestas a prueba por diferentes modelos teóricos permiten tener más razones o elementos de juicio para evaluar el porqué de la estructura de capital, pero a su vez hace más compleja una evaluación conjunta ya que se desconoce sus grados de incidencia, sus compatibilidades e interrelaciones. De ahí que existe un interés en la comunidad académica en estudiar los factores que inciden sobre la 
estructura de capital, para conocer bajo que precepto teórico se estaría tomando esta decisión.

\section{Marco de REFERENCIA}

En el campo internacional existe una predisposición a utilizar más recursos internos que deuda por parte de las empresas, al menos en los países desarrollados; también se observa que los 5 precios de las acciones reaccionan negativamente ante el anuncio de emisión de nuevas acciones y forma positiva cuando la emisión es de deuda. (Rivera, 2007, p.11)

CUADRO No. 4.

Algunos factores que influyen sobre el sobre el endeudamiento de acuerdo a diferentes modelos teóricos sobre la estructura de capital.

\begin{tabular}{lll}
\hline El apalancamiento aumenta con: & Modelo & Referencias \\
\hline Extensión de la asimetría de la información & Pecking order & Myers y Majluf (1984) \\
$\begin{array}{l}\text { Aumento de la rentabilidad } \\
\text { Disminución de la rentabilidad }\end{array}$ & Información asimétrica & Ross (1977) Ravid y Sarig (1991) \\
$\begin{array}{l}\text { La carencia de oportunidades de crecimiento } \\
\text { Aumento ene el flujo de tesorería libre }\end{array}$ & Agencia & Chang (1990) \\
$\begin{array}{l}\text { Disminución en flujos de tesorería libre } \\
\text { Disminución de los costos de investigación }\end{array}$ & Agencia & Jensen y Mecking (1976) \\
$\begin{array}{l}\text { Aumento en la importancia de reputación de la } \\
\text { administración }\end{array}$ & Agencia & Jesen (1986), Stulz (1990) \\
$\begin{array}{l}\text { El aplacamiento se correlaciona } \\
\text { positivamente con la probabilidad de } \\
\text { incumplimiento }\end{array}$ & Agencia & Myers y Majluf (1984) \\
$\begin{array}{l}\text { Las empresas con los registros más largos de } \\
\text { trayectoria, han dejado las probabilidades de } \\
\text { incumplimiento }\end{array}$ & Agencia & Harris y Raviv (1990) \\
\hline
\end{tabular}

FUENTE: Elaboración propia, teniendo como referencia a Harris y Raviv (1991)

Cuando se compara el endeudamiento entre empresas de una misma industria se observa que es más semejante que cuando se hace entre empresas de diferentes sectores. Hay sectores con bajos índices de endeudamiento, como el farmacéutico y electrónico, mientras otros como la siderúrgica y servicios públicos su endeudamiento es alto. (Rivera, 2007, p.12) En Colombia el nivel de endeudamiento del sector metalmecánica es alto (Rivera, 1998, p.223).

Rivera (1998) encontró el siguiente patrón de financiación en la empresa colombiana:

- La deuda a corto plazo es la principal fuente de financiación, seguido de las utilidades retenidas y por último la emisión de nuevas acciones.

- La estructura financiera está ligada a la actividad económica y tamaño de la empresa.
- Las pequeñas empresas utilizan como principal fuente de financiación los nuevos aportes de capital, las medianas recurren más a la deuda, mientras que las grandes empresas optan por la autofinanciación.

La estructura de capital de la mipyme en Colombia está conformada con un $55,36 \%$ de patrimonio y un $44,64 \%$ de deuda. Del total deuda, el 23,76\% es obligación financiera y el $23,22 \%$ proveedores, de acuerdo a una investigación realizada por la Superintendencia de Sociedades (2004) en el período 1999 -2003.

En este mismo estudio se observa que el sector manufacturero mantiene una alta y creciente concentración de deuda a corto plazo, destacándose la financiación con el sector financiero y proveedores. Ver cuadro 5. 
CUADRO No. 5 .

Indicadores de endeudamiento de mipymes sectores manufactureros en Colombia

\begin{tabular}{lcccc}
\hline Años & 1999 & 2000 & 2001 & 2002 \\
\hline Concentración pasivo corto plazo (\%) & 97,3 & 96,5 & 100 & 100 \\
\hline Endeudamiento con el sector financiero (\%) & 8,7 & 8,2 & 8,1 & 7,6 \\
\hline Endeudamiento con proveedores (\%) & 9,04 & 9,62 & 9,15 & 9,28 \\
\hline Tamaño de la muestra (número de empresas) & 726 & 726 & 726 & 726 \\
\hline Cuadro 5. Indicadores de endeudamiento de mipymes sectores manufactureros en Colombia \\
Fuente: Superintendencia de Sociedades, 2004, p. 27.
\end{tabular}

FUENTE: Superintendencia de Sociedades, 2004, p. 27.

FUNDES (2003) halla que las pymes utilizan como fuentes de financiación el capital propio en un 24 $\%$ y el otro $76 \%$ con recursos ajenos distribuidos así: crédito bancario $32 \%$, proveedores $18 \%$, créditos con corporaciones financieras $7 \%$, créditos con particulares $6 \%$,tarjetas de crédito 4 $\%$, cheque posfechados $4 \%$, el Estado $\%$, cooperativas y ONGs $1 \%$ y otros $2 \%$.

El nivel de endeudamiento del sector metalmecánica en Colombia aumentó en un 15,15 $\%$ en el período 2001-2005. Los índices de endeudamiento para estos años fue el siguiente:

$42,44 \%$ en el año $2001,42,69 \%$ en el año 2002 , $44,66 \%$ en el año $2003,47,32 \%$ en el año 2004 y $48,87 \%$ en el año 2005 (Portafolio, Nest \& Afine, 2006, p. 277)

A pesar de que no existe total unanimidad en los resultados de investigaciones en cuanto la relación que existe entre la deuda y algunos factores explicativos en el contorno mundial, se puede decir que el apalancamiento de la empresa tiende a subir cuando aumentan los activos fijos y/o el tamaño de la firma, y tiende a bajar cuando aumentan la rentabilidad de la firma, las oportunidades de crecimiento de la empresa, la volatilidad de la cuenta de resultados de la firma, los gastos de publicidad, investigación y desarrollo, la probabilidad de bancarrota $y / 0$ la originalidad del producto (Rivera, 2007, p.13).

En Colombia se ha encontrado una relación negativa entre el endeudamiento y la rentabilidad, la variabilidad de las utilidades, el costo de la deuda, la oportunidad de crecimiento, la edad de la firma los costos de insolvencia y la protección fiscal diferente a la deuda, y una relación positiva con el tamaño de la empresa y la razón de endeudamiento del período anterior. (Tenjo, López \& Samudio 2006; Sarmiento \& Salazar, 2005; Tenjo, 1996; Tenjo \& García, 1996) En las empresas medianas Tenjo (1996) y Tenjo y García (1996) hallan que el endeudamiento mantiene una relación negativa con la rentabilidad, la variabilidad de las utilidades, el costo de la deuda, la oportunidad de crecimiento y la edad de la empresa, y una relación positiva con el índice de endeudamiento del período anterior.

\section{Estudio de CAMPO DE LAS PYMES DEL SECTOR METALMECÁNICA DEL VALLE DEL Cauca}

La investigación se realiza con una muestra total de quince empresas pymes del Valle del Cauca, de la cuales cuatro son pequeñas y once medianas; de acuerdo a su estructura jurídica, siete son limitadas, seis sociedades anónimas, una en comandita por acciones y una en comandita simple. Al año 2000 sus edades oscilaban entre los 3 y 54 años, siendo el $40 \%$ empresas tradicionales (más de 22años), el $33 \%$ en proceso de consolidación (entre 6 y 15 años) y el $27 \%$ nuevas (menos de 6 años). Observar cuadro 6.

Se utiliza como fuente de información los estados financieros revelados en el período 2000-2006 disponible en: 
CUADRO No. 6.

Empresas representativas del sector metalmecánica

\begin{tabular}{lllll}
\hline \multicolumn{1}{c}{ Nombre } & Tamaño & \multicolumn{1}{c}{ Estructura Juridica } & Edad & Domicilio \\
\hline 1. Dimel Ingenieria S.A. & Pequeña & Sociedad Anónima & 12 años & Candelaria \\
2. Termomecánica Ltda. & Pequeña & Limitada & 11 años & Jamundi \\
3. Bea Andina Ltda. & Pequeña & Limitada & 13 años & Yumbo \\
4. Industrias Mecánicas de Colombia Vanegas & & Sociedad en Comandia por & & \\
\& Cía S. en C.A. & Pequeña & acciones & 29 años & Cali \\
5. Agrometal Urquijo Ltda. & Mediana & Limitada & 10 años & Cali \\
6. Octavio Gómez Cadavid e Hijos S en C & Mediana & Sociedad en Colombia & 10 años & Cali \\
7. Construcciones Metálicas Industriales Ltda & Mediana & Limitada & 9 años & Yumbo \\
8. Desarrollo Industrial del Aluminio S.A. & Mediana & Sociedad Anónima & 4 años & Yumbo \\
9. Industrias de Fijadores S.A. & Mediana & Sociedad Anónima & 4 años & Cali \\
10. Tissot S.A. & Mediana & Sociedad Anónima & 5 años & Cali \\
11. Sager S.A. & Mediana & Sociedad Anónima & 54 años & Cali \\
12. Colcadenas Ltda. & Mediana & Limitada & 39 años & Cali \\
13. Talleres de Metalización Tametco Ltda. & Mediana & Limitada & 29 años & Cali \\
14. Industrias Cato S.A. & Mediana & Sociedad Anónima & 31 años & Cali \\
15. Payan y Cia Ltda & Mediana & Limitada & 24 años & Cali \\
\hline
\end{tabular}

Nota: Para su clasificación se tomó como referencia los criterios estipulados por la ley 590 del 2000 y la ley 905 del 2004 , como, también, que hubiera sido creada antes del año 1999 y que estuviera funcionando por lo menos hasta finales del año 2004. Véase en el anexo1 las empresas objeto de estudio.

FUENTE: Elaboración propia

- La Base de Datos del Benchmark, de BPR Asociados Sales \& Credit Management, disponible en Univalle (http://www.BPR.com.co).

- La Base de Datos Superintendencia de Sociedades (http://www.supersociedades.gov.co).

\subsection{ESTRUCTURA FINANCIERA}

Las pymes del sector metalmecánica han venido aumentando su nivel de endeudamiento en los últimos años del periodo 2000-2006; este índice es moderado para las medianas $(31,8 \%$ en promedio) y bastante alto para las pequeñas (por encima del 90\%). Se destaca una alta concentración de endeudamiento a corto plazo ( $76,8 \%$ en promedio), y en orden jerárquico, de mayor a menor promedio, hacen uso de las siguientes tipos de deuda: acreedores varios $(31,9 \%)$, obligaciones financieras $(25,2 \%)$, proveedores $(18,7 \%)$, impuestos $(8,9 \%) \mathrm{y}$ obligaciones laborales $(7,5 \%)$.

Al comparar la forma como se financian con recursos ajenos la pequeña y mediana empresa, se observa que la primera obtiene más recursos con las entidades financieras, se apalanca mucho más con acreedores varios y con impuestos; mientras que la segunda tiene una mayor concentración de deuda a corto plazo, recurre a un mayor endeudamiento con proveedores y utiliza en mayor proporción las obligaciones laborales como fuente de financiación. Ver cuadro 7.

\subsection{FaCtores EXPLICATIVOS DE LA ESTRUCTURA DE CAPITAL}

\subsubsection{Modelo econométrico}

Para conocer los factores influyentes en la estructura de capital de las pymes del sector metalmecánica del Valle del Cauca se utiliza una modelación econométrica de Datos de Panel para los años 2001 2006.

En un modelo de Datos de Panel se considera tanto la dimensión temporal como la estructural, lo que permite capturar la heterogeneidad de los agentes económicos, e incorpora el análisis dinámico (Greene, 1999, capítulo 14). Este modelo tiene dos formas básicas de aplicarse:

La primera, es con efectos fijos, donde se pretende captar las diferencias que existen entre las unidades de análisis por características propias de cada una de ellas, a través del término constante, y se representa así:

Yit $=\mathrm{mi}+\mathrm{bXit}+\mathrm{e}$ it 
CUADRO No. 7.

Indicadores de endeudamiento de pymes del sector metalmecánica de la ciudad de Cali

\begin{tabular}{|c|c|c|c|c|c|c|c|c|}
\hline & & \\
\hline & & 2000 & 2001 & 2002 & 2003 & 2004 & 2005 & 2006 \\
\hline \multirow{3}{*}{ Nivel de Endurecimiento \% } & Pequeñas & 92,5 & 103,3 & 95,8 & 90,1 & 88,2 & 93,2 & 94,2 \\
\hline & Medianas & 22,6 & 29,9 & 26,0 & 32,7 & 34,9 & 36,6 & 40,0 \\
\hline & Pymes & 30,7 & 37,4 & 33,4 & 38,3 & 39,5 & 40,9 & 43,9 \\
\hline \multirow{3}{*}{$\begin{array}{l}\text { Concentración Endeudamiento } \\
\text { Corto Plazo \% }\end{array}$} & Pequeñas & 55,8 & 66,8 & 67,1 & 54,6 & 45,3 & 47,6 & 57,3 \\
\hline & Medianas & 86,0 & 79,5 & 80,1 & 83,2 & 85,3 & 83,6 & 82,6 \\
\hline & Pymes & 75,4 & 75,9 & 76,1 & 76,6 & 77,5 & 77,5 & 78,7 \\
\hline \multirow{3}{*}{$\begin{array}{l}\text { Concentración Endeudamiento } \\
\text { Entidades Financieras \% }\end{array}$} & Pequeñas & 33,2 & 27,7 & 29,7 & 35,2 & 36,7 & 40,6 & 33,7 \\
\hline & Medianas & 38,9 & 17,7 & 28,1 & 16,9 & 20,7 & 19,6 & 20,6 \\
\hline & Pymes & 36,9 & 20,5 & 28,6 & 21,1 & 23,8 & 23,1 & 22,7 \\
\hline \multirow{3}{*}{$\begin{array}{l}\text { Concentración Endeudamiento } \\
\text { Proveedores \% }\end{array}$} & Pequeñas & 9,6 & 7,6 & 5,3 & 8,2 & 10,8 & 10,8 & 13,5 \\
\hline & Medianas & 25,2 & 16,6 & 23,8 & 15,0 & 18,7 & 24,4 & 28,3 \\
\hline & Pymes & 19,8 & 14,1 & 18,1 & 13,5 & 17,1 & 22,1 & 26,0 \\
\hline \multirow{3}{*}{$\begin{array}{l}\text { Concentración Endeudamiento } \\
\text { Obligaciones Laborales \% }\end{array}$} & Pequeñas & 4,9 & 4,6 & 5,0 & 6,6 & 6,7 & 6,5 & 5,5 \\
\hline & Medianas & 8,1 & 8,7 & 7,6 & 8,9 & 9,2 & 8,2 & 6,6 \\
\hline & Pymes & 7,0 & 7,6 & 6,8 & 8,4 & 8,7 & 7,9 & 6,4 \\
\hline \multirow{3}{*}{$\begin{array}{l}\text { Concentración Endeudamiento } \\
\text { Impuestos \% }\end{array}$} & Pequeñas & 12,1 & 11,6 & 9,5 & 11,1 & 10,6 & 9,5 & 12,6 \\
\hline & Medianas & 8,6 & 12,5 & 10,8 & 8,8 & 6,3 & 5,7 & 5,9 \\
\hline & Pymes & 9,8 & 12,3 & 10,4 & 9,3 & 7,1 & 6,3 & 6,9 \\
\hline \multirow{3}{*}{$\begin{array}{l}\text { Concentración Endeudamiento } \\
\text { Otras Deudas \% }\end{array}$} & Pequeñas & 39,6 & 48,3 & 50,1 & 38,6 & 35,0 & 31,5 & 34,1 \\
\hline & Medianas & 15,7 & 31,9 & 23,4 & 36,4 & 32,2 & 30,3 & 30,1 \\
\hline & Pymes & 24,1 & 36,5 & 31,6 & 36,9 & 32,7 & 30,5 & 30,7 \\
\hline
\end{tabular}

Fuente: Elaboración propia.

Donde:

i es la empresa.

t es el período de tiempo.

Yit es el nivel de endeudamiento a largo plazo. ii es el efecto fijo adjudicadle a cada empresa, y es constante a lo largo del tiempo.

â es el vector de $\mathrm{k}$ parámetros estimados (una para cada variable explicativa).

Xit es la observación de la i-ésima empresa en el momento t para las $\mathrm{k}$ variables explicativas. åit es el término de error aleatorio.

La segunda forma es con efectos aleatorios; aquí se considera que el efecto de cada empresa se distribuye de forma aleatoria. Por lo tanto los efectos individuales de todas las unidades se adicionan al error aleatorio general de todo el modelo; y se expresa de la siguiente manera:

Yit $=a+b X i t+(m i+e$ it $)$

Donde:

á es el término constante para todas las empresas. ì es el efecto aleatorio que permitiría distinguir el efecto de cada unidad en el panel. åit es el término de error aleatorio del modelo general.

Las otras convenciones son iguales a las definidas para el anterior modelo.

Las variables explicativas que componen el modelo son:

- La volatilidad de la utilidad antes de interés e impuestos (UAII), medida mediante su desviación estándar.

- La protección fiscal diferente a la deuda, calculada así: [depreciación / UAII]i,t

- La oportunidad de crecimiento, calculada así: [(activos - capital $+\ln$ activo fijo $) /$ activos $]$ i, $t$ $[($ activos - capital $+\ln$ activo fijo $) /$ activos $]$ i,t$1^{1}$

- La rentabilidad [UAII / activos]i,t

- La retención de utilidades, determinado por el índice [utilidades retenidas / utilidad después de intereses e impuestos]i,t

- La tangibilidad, hallada mediante el siguiente cociente: [activos fijos tangibles / activos]i,t

- El tamaño de la firma, medido por el logaritmo natural de las ventas $[\ln$ ventas]i,t

El logaritmo natural del activo fijo se usa en reemplazo del valor de mercado de capital, debido que estas empresas no cotizan en bolsa de valores. 
- Estructura de capital de corto plazo, mediante el índice [deuda a corto plazo / activos]i,t

- Edad de la empresa [años en actividad]i,t

- Costo de la deuda [gastos financieros / pasivos]

Todas las variables explicativas son de carácter contable - financiero.

\subsubsection{Proceso y resultados de las estimaciones}

Se estima modelos tanto para el conjunto de las pymes del sector metalmecánica, como, también, en forma separada para el grupo de pequeñas y de medianas empresas.

El proceso de estimación se inició con la medición de las variables explicativas, después se procedió a correr los modelos de Datos de Panel en el programa estadístico Stata 9.0. Se utilizó el Test de Hausman ${ }^{2}$ para establecer cuál de los modelos se ajusta mejor a los datos, si estos corresponden a un modelo con efectos fijos o con efectos aleatorios. Los errores estándar de los coeficientes son robustos, es decir, que están ajustados para corregir heterocedasticidad y autocorrelación.

Al estimar por separado las empresas pequeñas, medianas y pymes se observa que en la primera los datos se ajustan mejor a un modelo de efectos fijos y en las dos últimas el modelo más apropiado es el de efectos aleatorios, según el Test de Hausman. Las variables que son estadísticamente significativas y sus relaciones con el endeudamiento a largo plazo son:

Para las pequeñas empresas, la estructura de capital a corto plazo (-) y la edad (+); para las medianas empresas, la volatilidad de la utilidad antes de intereses e impuestos $(+)$, los activos tangibles $(+)$, el tamaño de la firma (-) y la edad $(+)$; mientras para las pymes, la protección fiscal diferente a la deuda (-), la oportunidad de crecimiento $(+)$ y la rentabilidad (-), como se muestra en el cuadro 8.

CUADRO No. 8.

Estimación para las pequeñas, medinas y pymes empresas del sector metalmecánica.

\begin{tabular}{|c|c|c|c|c|c|c|}
\hline & \multicolumn{2}{|c|}{$\begin{array}{l}\text { Empresas PEQUEÑAS } \\
\text { Variable dependiente: } \\
\text { Endeudamiento a Largo Plazo }\end{array}$} & \multicolumn{2}{|c|}{$\begin{array}{l}\text { Empresas MEDIANAS } \\
\text { Variable dependiente: } \\
\text { Endeudamiento a Largo Plazo }\end{array}$} & \multicolumn{2}{|c|}{$\begin{array}{l}\text { Empresas PYMES } \\
\text { Variable dependiente: } \\
\text { Endeudamiento a Largo Plazo }\end{array}$} \\
\hline $\begin{array}{l}\text { VARIABLE } \\
\text { INDEPENDIENTE }\end{array}$ & E. FIJOS & $\begin{array}{c}\text { E. } \\
\text { ALEATORIOS }\end{array}$ & E. FIJOS & $\begin{array}{c}\text { E. } \\
\text { ALEATORIOS }\end{array}$ & E. FIJOS & $\begin{array}{c}\text { E. } \\
\text { ALEATORIOS }\end{array}$ \\
\hline \multirow{3}{*}{$\begin{array}{l}\text { Constante } \\
\text { Estructura capital a } \\
\text { corto plazo } \\
\text { Volatilidad de las } \\
\text { UAll }\end{array}$} & $\begin{array}{c}3.614678^{\star *} \\
(0.8697929)\end{array}$ & $\begin{array}{c}2.91991^{* *} \\
(1.36038)\end{array}$ & $\begin{array}{r}-0.0797588 \\
(0.0707966)\end{array}$ & $\begin{array}{l}-0.0663811^{\star *} \\
(0.0162472)\end{array}$ & $\begin{array}{l}0.2956638 \\
(0.2310154)\end{array}$ & $\begin{array}{c}0.084898 \\
(0.1239868)\end{array}$ \\
\hline & $\begin{array}{c}-0.4948247^{* \star *} \\
(0.0675709)\end{array}$ & $\begin{array}{l}-0.3136355^{\star \star} \\
(0.1287039)\end{array}$ & $\begin{array}{r}-0.2545375 \\
(0.173284)\end{array}$ & $\begin{array}{l}-0.03355393 \\
(0.0838357)\end{array}$ & $\begin{array}{r}-0.3834517^{* *} \\
(0.0960855)\end{array}$ & $\begin{array}{c}0.0540351 \\
(0.127034)\end{array}$ \\
\hline & $\begin{array}{c}1.67 \mathrm{e}-09 \\
(5.44 \mathrm{e}-07)\end{array}$ & $\begin{array}{c}5.77 \mathrm{e}-08 \\
(6.20 \mathrm{e}-07)\end{array}$ & $\begin{array}{c}-3.17 \mathrm{e}-08 \\
(1.83 \mathrm{e}-08)\end{array}$ & $\begin{array}{l}6.81 \mathrm{e}-08^{* *} \\
(2.29 \mathrm{e}-08)\end{array}$ & $\begin{array}{l}2.10 \mathrm{e}-08 \\
(7.20 \mathrm{e}-08)\end{array}$ & $\begin{array}{c}4.31 \mathrm{e}-08 \\
(6.59 \mathrm{e}-08)\end{array}$ \\
\hline \multirow{3}{*}{$\begin{array}{l}\text { Protección fiscal } \\
\text { diferente a la deuda } \\
\text { Oportunidad de } \\
\text { Crecimiento } \\
\text { Rentabilidad }\end{array}$} & $\begin{array}{l}-0.0255285 \\
(0.0253941)\end{array}$ & $\begin{array}{l}-0.0323131 \\
(0.0289626)\end{array}$ & $\begin{array}{c}-3.108296^{\star *} \\
(1.303897)\end{array}$ & $\begin{array}{l}-5.099669 \\
(5.363841)\end{array}$ & $\begin{array}{l}-0.0318814^{*} \\
(0.0152361)\end{array}$ & $\begin{array}{c}-0.1058894^{\star *} \\
(0.0171015)\end{array}$ \\
\hline & $\begin{array}{l}-0.3063812 \\
(0.154751)\end{array}$ & $\begin{array}{l}-0.2273398 \\
(0.2101591)\end{array}$ & $\begin{array}{l}0.1748823^{*} \\
(0.0855944)\end{array}$ & $\begin{array}{l}0.0714756 \\
(0.112448)\end{array}$ & $\begin{array}{l}0.2733911^{* *} \\
(0.0341972)\end{array}$ & $\begin{array}{l}-0.1158944^{\star \star} \\
(0.0297462)\end{array}$ \\
\hline & $\begin{array}{c}-0.4378406 \\
(0.2404727)\end{array}$ & $\begin{array}{c}-0.0882787 \\
(0.2185405)\end{array}$ & $\begin{array}{l}0.1748823^{*} \\
(0.0772736)\end{array}$ & $\begin{array}{c}0.0419825 \\
(0.1647757)\end{array}$ & $\begin{array}{l}-03188664^{\star *} \\
(0.0764441)\end{array}$ & $\begin{array}{l}-0.2242035^{\star \star} \\
(0.1003596)\end{array}$ \\
\hline \multirow{2}{*}{$\begin{array}{l}\text { Retención de } \\
\text { utilidades } \\
\text { Activos tangibles }\end{array}$} & $\begin{array}{c}-0.0051354 \\
(0.0064539)\end{array}$ & $\begin{array}{l}-0.0023087 \\
(0.0063679)\end{array}$ & $\begin{array}{r}(5.60 \mathrm{e}-06) \\
(0.0001365)\end{array}$ & $\begin{array}{r}-0.0003561 \\
(0.0002974)\end{array}$ & $\begin{array}{r}0.00001516 \\
(0.0002326)\end{array}$ & $\begin{array}{l}-0.0000687 \\
(0.0003093)\end{array}$ \\
\hline & $\begin{array}{r}-1.101394 \\
(0.5815728)\end{array}$ & $\begin{array}{l}-0.8067372^{\star *} \\
(0.0705021)\end{array}$ & $\begin{array}{r}0.0414984 \\
(0.0380482)\end{array}$ & $\begin{array}{c}0.1940635^{*} \\
(0.0823206)\end{array}$ & $\begin{array}{r}-0.0896772 \\
(0.1376603)\end{array}$ & $\begin{array}{r}-0.0151233 \\
(0.0861853)\end{array}$ \\
\hline Tamaño de la forma & $\begin{array}{l}-0.1413007 \\
(0.07841)\end{array}$ & $\begin{array}{l}-0.24036^{* *} \\
(0.0934846)\end{array}$ & $\begin{array}{r}0.0026426 \\
(0.0018465)\end{array}$ & $\begin{array}{c}-0.003848^{*} \\
(0.0012382)\end{array}$ & $\begin{array}{l}-0.0060427^{*} \\
(0.0030295)\end{array}$ & $\begin{array}{r}-0.0013109 \\
(0.0031771)\end{array}$ \\
\hline Costo de la ducha & $\begin{array}{c}-0.0101771 \\
(0.0742233)\end{array}$ & $\begin{array}{c}-0.1351407 \\
(0.1755551)\end{array}$ & $\begin{array}{r}0.0003795 \\
(0.0002142)\end{array}$ & $\begin{array}{c}0.0000663 \\
(0.0001574)\end{array}$ & $\begin{array}{r}0.0005006^{*} \\
(0.0002379)\end{array}$ & $\begin{array}{l}-0.0001175 \\
(0.000215)\end{array}$ \\
\hline Edad & $\begin{array}{l}-0.0393553^{\star \star} \\
(0.0085646)\end{array}$ & $\begin{array}{r}0.0544569^{\star \star \star} \\
(0.0104607)\end{array}$ & $\begin{array}{l}0.0089848 \\
(0.0050824)\end{array}$ & $\begin{array}{l}0.0031165^{\star \star} \\
(0.0010306)\end{array}$ & $\begin{array}{c}0.003772 \\
(0.0084991)\end{array}$ & $\begin{array}{c}0.0038945 \\
(0.0043333)\end{array}$ \\
\hline \multirow{3}{*}{$\begin{array}{l}\mathrm{R}^{2} \\
\text { No. De } \\
\text { Observaciones } \\
\text { No. de grupos }\end{array}$} & -0.2093 & 0.9562 & 0.0053 & 0.3911 & 0.0006 & 0.2153 \\
\hline & 24 & 24 & 66 & 66 & 90 & 90 \\
\hline & 4 & 4 & 11 & 11 & 15 & 15 \\
\hline $\begin{array}{l}\text { Test de Hausman } \\
\left(\mathrm{Chi}^{2}\right)\end{array}$ & \multicolumn{2}{|l|}{21.01} & \multicolumn{2}{|c|}{14.88} & \multicolumn{2}{|c|}{-9.23} \\
\hline
\end{tabular}

Nota: $* * *$ Significativa al $1 \%, * *$ Significativa al 5\%, $*$ Significativa al 10\%. Errores estándar robustos entre paréntesis. Fuente: Elaboración propia.

Test de Hausman

Ho: âefectos aleatorios - âefectos fijos $=0$, el estimador de efectos aleatorios es eficiente y el de efectos fijos es consistente. Ha: âefectos aleatorios - âefectos fijos " 0 el estimador de efectos fijos es consistente y el de efectos aleatorios es ineficiente. 


\section{ANÁLISIS y COMPARACIÓN DE RESULTADOS}

El nivel endeudamiento de la pyme ha mantenido la tendencia creciente del sector metalmecánica en Colombia, aunque su relación deuda a activo total es más baja. (Portafolio, Nest \& Afine, 2006, p. 277)

El alto endeudamiento de la pequeña empresa corrobora lo hallado por FUNDES (2003) para la pymes en Colombia, y la alta concentración de deuda de corto plazo por parte de la mediana empresa sigue la tendencia de la industria en Colombia de mantener una alta concentración de deuda a corto plazo. Rivera (1998) y Superintendencia de Sociedades (2004).

En el cuadro 9 se resume los principales factores determinantes de la estructura de capital de las pymes del sector metalmecánica del Valle del Cauca y su relaciones positiva (+) o negativa (-) frente un aumento del endeudamiento a largo plazo, y se compara con resultados de investigaciones realizadas en el ámbito internacional y en Colombia.

CUADRO No.9.

Variables determinantes de la estructura de capital del sector pymes metalmecánica del Valle del Cauca

\begin{tabular}{l|c|c|c|c|c}
\hline VARIABLE INDEPENDIENTE & Pequeñas & Medianas & Pymes & $\begin{array}{c}\text { Evidencia } \\
\text { internacional }\end{array}$ & $\begin{array}{c}\text { Evidencia en } \\
\text { Colombia }\end{array}$ \\
\hline Estructura de capital a corto plazo & - & & & & \\
\hline Volatilidad de UAll & & + & & & \\
\hline Protección fiscal diferente a la deuda & & & - & $-+^{\mathrm{n} 1}$ & - \\
\hline Oportunidad de crecimiento & & & + & - & - \\
\hline Rentabilidad & & & - & - & - \\
\hline Activos tangibles & & + & & + & $-+^{\mathrm{n} 2}$ \\
\hline Tamaño de la firma & & - & & + & + \\
\hline Edad & + & + & & & - \\
\hline
\end{tabular}

n1 Existe evidencias opuestas: relación negativa (Kim y Sorense, 1986) y relación positiva (Bradley, Jarrell y Kim, 1984). n2 Existe evidencias opuestas: relación negativa (Sarmiento y Salazar, 2005) y relación positiva (Tenjo, et al., 2006).

Fuente: Elaboración propia.

De las diez variables exploradas, tres son significativas para las pymes, dos para las pequeñas y cuatro para las medianas, y ninguna es explicativa para los tres grupos de empresas; tan solo una es explicativa tanto para pequeñas como medianas empresas, sin existir contradicción en la relación de la variable determinante y el endeudamiento a largo plazo.

En la pequeña empresa el aumento del endeudamiento a corto plazo reduce la deuda a largo plazo; lo que seguramente podría significar que se utilizan recursos de corto plazo para emprender proyectos de largo plazo, o deuda de largo plazo para financiar capital de trabajo.

El aumento de la protección fiscal diferente a la deuda disminuye el uso de la deuda de largo plazo como fuente de financiación de las pymes; lo que podría interpretarse como una reacción a utilizar menos deuda a medida que se pierda la posibilidad de sacar beneficio fiscal por el endeudamiento. Esta tendencia va acorde con el planteamiento de la teoría del trade off(Deangelo \& Masulis, 1980).

La volatilidad de la utilidad antes de intereses e impuestos afecta de forma directa el endeudamiento a largo plazo de las medianas empresas, contrario a la evidencia internacional y en Colombia. Lo que se puede interpretar que el aumento de la probabilidad de quiebra, resultado de la variación de las utilidades operacionales, no influye negativamente en la consecución de recursos ajenos de largo plazo.

La oportunidad de crecimiento para las pymes, contrario a los resultados de otras investigaciones en el campo internacional y en Colombia, ha influido positivamente para el aumento del endeudamiento a largo plazo; lo que permite presumir la inexistencia de costos de agencia y de información asimétrica por posibles conflictos de interese entre propietarios y prestamistas, quizás porque las condiciones contractuales de la deuda evita que se presenten situaciones de subinversión. 
Si se considera que una disminución de la rentabilidad reduce los flujos de tesorería, un aumento del endeudamiento cuando baja la rentabilidad, como sucede con las pymes, debería interpretarse como una segunda opción que utilizan las empresas cuando se reducen sus fondos internos, tal como lo plantea la teoría del pecking order (Myers \& Majluf,1984).

Los activos fijos tangibles influyen de forma positiva sobre la estructura de capital de las empresas medianas, por el mayor respaldo que estos representan en caso de una eventual caída a un proceso de quiebra y porque reduce los posibles costos de agencia relacionados con la deuda (Jensen \& Meckling, 1976). La evidencia empírica internacional corrobora esta tendencia.

En la mediana empresa se observa que el endeudamiento a largo plazo disminuye a medida que aumenta el tamaño de la empresa, contrario a lo que muestra la evidencia empírica internacional y nacional. Esto no solamente se refleja al interior de la mediana empresa, sino cuando se compara la pequeña con la mediana, se encuentra un mayor endeudamiento a largo plazo en la primera.

La mayor edad de la empresa pequeña y mediana influye en el aumento de deuda a largo plazo, quizás porque su mayor madurez reduce la probabilidad de incumplimiento como lo plantea la teoría de agencia (Diamond, 1989).

\section{Conclusiones}

La industria metalmecánica es mayoritariamente pyme, y su contribución al PIB y generación de empleo es altamente significativa. Pero en una economía globalizada e integrada, las posibilidades de supervivencia y crecimiento dependen de sus gestiones para mejorar la competitividad; lo que demanda recursos financieros.
En la literatura financiera la consecución de recursos depende de una gran variedad de factores, algunos de ellos influyentes sobre el valor de la empresa, que han sido evaluados en modelos teóricos y trabajos de campo.

Este trabajo realiza una prueba con el sector pyme de metalmecánica en el Valle del Cauca en el periodo 2000 - 2006 para identificar la estructura financiera y los factores determinantes de la estructura de capital, utilizando como herramienta metodológica el análisis económicofinanciero y un modelo de Datos de Panel.

En esta investigación se encontró que existen diferencias en la estructura financiera y los factores relevantes que explican el endeudamiento a largo plazo de la pequeña, mediana y pyme, a pesar de la similitud de su actividad y de estar en una misma región.

La pequeña empresa se endeuda más que la mediana, y utiliza como principales fuentes de recursos ajenos los préstamos con acreedores varios y las obligaciones financieras; mientras que la mediana presenta una mayor concentración de deuda a corto plazo y se destaca la financiación con proveedores.

Los factores determinantes de la estructura da capital para las pymes y su relación positiva $(+)$ o negativa (-) con relación al endeudamiento a largo plazo son: En las pequeñas, la estructura de capital a corto plazo (-) y la edad (+). En las medianas, la volatilidad de la utilidad antes de intereses e impuestos $(+)$, los activos tangibles (+) el tamaño de la firma $(-)$ y la edad $(+)$. En la pyme, la protección fiscal diferente a la deuda (-), la oportunidad de crecimiento $(+)$ y la rentabilidad (-).

Cuando los resultados son cotejados con modelos teóricos y evidencia empíricas, no se prueba que se ajuste a alguno de ellos en forma particular. 


\section{REFERENCIAS BIBLIOGRÁFICAS}

Altman, E. (1984). A further empirical investigation of the bankruptcy cost question. The Journal of Finance, 39 (4), September, pp.1.067-1.089.

Bradley, M.;Jarrell, G. y Kim, H. (1984). On the existence of an optimal capital structure: theory and evidence. The Journal of Finance, 39 (3), July, pp. 857-880.

Brealey, R. y Myers, S. (2003). Principios de Finanzas Corporativas, septima edicion. MadridEspaña: McGraw- Hill.

Chang, C. (1990): The dinamic structure of optimal debt contracts. Journal of Economic Theory, 52 (1), October, pp. 68-86.

DANE (2007). Encuesta Anual Manufacturera 2005. Recuperado el 17 de julio de 2007, de http:// www.dane.gov.co/index.php?option $=$ com_content\&task $=$ category\&sectionid $=17 \& \mathrm{id}=43 \&$ Itemid $=154$

DeAngelo, H. y Masulis, R. (1980). Optimal capital structure under corporate and personal taxation. Journal of Financial Economics, 8, March, pp. 3 - 29.

Diamond, D. (1989). Reputation acquisition in debt markets. Journal of Political Economy, 97 (4),

August, pp. $828-862$.

FUNDES (2003). La realidad de la pyme colombiana. Recuperado el 25 de octubre de 2006, de http:// www.cta.org.co/maps/ doc/La realidad de la pyme colombiana fundes.ppt.

Greene, W. (1999). Análisis Econométrico, Tercera Edicon. Madrid: Prentice Hall.

Harris, M. y Raviv, A. (1.990). Capital structure and the informational role of debt. The Journal of

Finance, 45 (2), June, pp. $321-349$.

Harris, M. Raviv, A. (1991). The Theory of Capital Structure. The Journal of Finance, 46 (1), March, pp 297-355.

Hirsleifer, D. y Thakor, A. (1989). Managerial reputation, project choice and debt. Working paper No. 14-89, Anderson Graduate School of Management at UCLA.

Jensen, M. y Meckling, W. (1976). Theory of the firm: managerial behavior, agency cost and ownership structure. Journal of Financial Economics, 3, October, pp. 305 - 360.

Jensen, M. (1986). Agency costs of free cash flow, corporate finance, and takeovers. The American Economic Review, 76 (2), May, pp. 323 - 329.

Kim, E. (1978). A mean variance theory of optimal capital structure and corporate debt capacity. The Journal of Finance, 32 (1), March, pp. $45-63$.

Kim, W. y Sorense, E. (1986). Evidence on impact of the agency costs of debt on corporate debt policy. Journal of Financial and Quantitative Analysis, 21 (2), June, pp. 131-144.

Miller M. y Modigliani, F. (1963). Corporate income taxes and the cost of capital: a correction. The American Economic Review, 53, June, pp. 433 - 443

Modigliani, F. y Miller M. (1958). The cost of capital, corporation finance and the theory of investment. The American Economic Review, 48, June, pp. 261 - 297.

Myers, S. (1984). The capital structure puzzle. The Journal of Finance, 39 (3), July, pp. 575 - 591. Myers, S. y Majluf, N. (1984). Corporate financing and investment decisions when firms have information that investors do not have. Journal of Financial Economics, 13, july, pp.187-221.

Portafolio, Nest y Afine (2006). El gran libro de las pymes; información financiera. Bogotá: Casa editorial El Tiempo.

Rajan, R. y Zingales, L. (1995). ¿What do we know about capital structure? Some evidence from international data. The Journal of Finance, 50 (5), December, pp. 1.421 - 1460.

Ravid, S. y Sarig, O. (1991). Financial signaling by committing to cash outflows. Journal of Financial and Quantitative Analysis, 26 (2), June, pp. 165 - 189.

Rivera, J. (1998). Factores determinantes de la estructura de capital de las grandes empresas industriales en Colombia. Tesis doctoral. España: Universidad Autónoma de Madrid.

(2007, junio). Estructura financiera y factores determinantes de la estructura de capital de la pyme del sector confecciones del Valle del Cauca en el período 2000 - 2004. Documento presentado en el IV simposio Nacional y I Internacional de docentes en finanzas. Cartagena de Indias, Colombia.

Ross, S. (1977). The determination of financial structure: the incentive-signaling approach. The Bell Journal of Economics, 8 (1), Spring, pp. 23 - 40. 
Sarmiento, R. y Salazar M. (2005). La estructura de financiamiento de las empresas: una evidencia teórica y econométrica para Colombia. 1997-2004. Bogotá: Facultad de Ciencias Económicas y Administrativas, Pontificia Universidad Javeriana.

Superintendencia de Sociedades (2007a). Boletín Estadístico - Estados Financieros 2006. Recuperado el día 17 de julio de 2007 de http://www.supersociedades.gov.co/ss/drvisapi.dll?MIval $=$ sec \&dir $=329$

Superintendencia de Sociedades (2007b). Informes Especiales. Las mipymes en Colombia. Recuperado el 17 de julio de 2007 de http://www.supersociedades.gov.co/ss/drisapi.dll/Las\%20 mipymes\%en $20 \%$ colombia.pdf

Superintendencia de Sociedades (2004). Las mipymes en Colombia: evolución, desarrollo y fomento (1999-2003). Recuperado el 25 de octubre de 2006, de http://www.supersociedades.gov.co/ss/ drisapi.dll/Las\%20mipymes\%en20\%colombia.pdf

Stulz, R. (1990). Managerial discretion and optimal financing polices. Journal of Financial Economics, 26, pp. 3-27.

Tenjo, F., López, E. y Zamudio, N. (2006). Determinantes de la estructura de capital de las empresas colombianas (1996 - 2002). Borradores de Economía No. 380. Bogota: Subgerencia de estudios del Banco de la República de Colombia.

Tenjo, F. (1996). Restricciones financieras, comportamiento de las empresas manufactureras y perspectivas para el desarrollo de mercados de capitales en Colombia. Cali: Mimeo, Misión de Estudios del Mercado de Capitales, pp. 1-48.

Tenjo, F. y García, G. (1996). Desarrollo financiero y estructura de capital de las empresas. Banca y Finanzas, No. 40, abril-junio, pp. 5-34. 